\title{
Integration of Molecular Analysis, Cutting-edge Mouse Genetic Models and Proton Therapy to Improve Outcomes for Glioma Patients
}

\author{
Soma Sengupta ${ }^{1} \bullet$ Luke E. Pater $^{2} \bullet$ Daniel Pomeranz Krummel $^{1} \bullet$ \\ Bruce J. Aronow ${ }^{3} \bullet$ Yoshihisa Hirota $^{4,5} \bullet$ Timothy N. Phoenix ${ }^{6,7} \bullet$ \\ Atsuo T. Sasaki $4,8,9,10$
}

${ }^{1}$ Department of Neurology and Rehabilitation Medicine, University of Cincinnati College of Medicine, Cincinnati, OH, USA; ${ }^{2}$ Department of Radiation Oncology at the University of Cincinnati College of Medicine, Cincinnati, OH, USA; ${ }^{3}$ Department of Biomedical Informatics, Cincinnati Children's Hospital Medical Center, Cincinnati, Ohio, USA; ${ }^{4}$ Division of Hematology and Oncology, Department of Internal Medicine, University of Cincinnati College of Medicine, Cincinnati, OH, USA; ${ }^{5}$ Department of Bioscience and Engineering, College of Systems Engineering and Science, Shibaura Institute of Technology, Fukasaku, Minuma-ku, Saitama, Japan; ${ }^{6}$ Division of Pharmaceutical Sciences, James L. Winkle College of Pharmacy, University of Cincinnati, Cincinnati, Ohio, USA; ${ }^{7}$ Research in Patient Services, Cincinnati Children's Hospital Medical Center (CCHMC), Cincinnati, Ohio; USA; ${ }^{8}$ Department of Cancer Biology, University of Cincinnati College of Medicine, OH, USA; ${ }^{9}$ Department of Neurosurgery, Brain Tumor Center at UC Gardner Neuroscience Institute, Cincinnati, OH, USA; ${ }^{10}$ Institute for Advanced Biosciences, Keio University, Tsuruoka, Japan

Authors for correspondence: Soma Sengupta, Department of Neurology and Rehabilitation Medicine, University of Cincinnati College of Medicine, Cincinnati, $\mathrm{OH}$, USA. Email: sengupsm@ucmail.uc.edu; Luke E. Pater, Department of Radiation Oncology at the University of Cincinnati Barrett Cancer Center, Cincinnati, OH, USA.

Email: paterl@ucmail.uc.edu; Timothy N. Phoenix, Division of Pharmaceutical Sciences, James L. Winkle College of Pharmacy, University of Cincinnati, Cincinnati, Ohio, USA. Email: phoenity@ucmail.uc.edu; Atsuo T. Sasaki, Division of Hematology and Oncology, Department of Internal Medicine, University of Cincinnati College of Medicine, Cincinnati, OH, USA. Email: atsuo.sasaki@uc.edu

Doi: https://doi.org/10.36255/exonpublications.gliomas.2021.chapter5

In: Gliomas. Debinski W (Editor). Exon Publications, Brisbane, Australia. ISBN: 978-0-6450017-4-7; Doi: https://doi.org/10.36255/exonpublications.gliomas.2021

Copyright: The Authors.

License: This open access article is licenced under Creative Commons Attribution-NonCommercial 4.0 International (CC BY-NC 4.0) https://creativecommons.org/licenses/by-nc/4.0/ 


\begin{abstract}
Despite recent advances in general cancer treatment, glioblastoma remains among the most lethal of human malignancies. Even with aggressive multimodal radiation and chemotherapy after surgery, glioblastoma recurs with a bleak prognosis. Decades of research focused on strategies such as increasing radiation sensitivity and interference with oncogenic signal transduction have yielded only incremental improvements at best. This is due in part to the radioresistance of glioblastoma and molecular heterogeneity of tumor cells. We hypothesize is that the development of more effective glioblastoma therapies will require: (i) a more accurate molecular analysis of glioblastoma so as to predict response to therapy; (ii) better genetically engineered mouse models, which can faithfully recapitulate human glioblastoma and the tumor microenvironment to test new approaches and (iii) development and application of more accurate and focused methods to deliver sustained high energy particles to glioblastoma tumor sites. This chapter describes the current state-of-the-art molecular analysis approaches, latest in glioma mouse modelling, and advances in the application of proton therapy treatment and research. By integrating basic and clinical research with cutting-edge technologies, a mechanistic understanding of glioblastoma therapy resistance and pathogenesis and the development of new therapeutics to overcome the therapeutic resistance of glioblastoma will be advanced.
\end{abstract}

Keywords: brain tumor-associated edema; in utero electroporation-based glioma mouse models; mycophenolic acid; proton beam therapy; single-cell RNA sequence

\title{
INTRODUCTION
}

Glioblastoma is a WHO Grade IV primary brain cancer with an abysmal prognosis (1). The current gold standard initial treatment approach is a gross resection of the tumor guided by the use of 5-Aminolevulinic Acid (5-ALA), a porphyrin precursor, to identify the infiltrative margins (2). Such emerging imaging approaches combined with advances in surgical techniques have improved surgical outcomes, but the undetected residual microscopic disease remains a significant problem. Gross surgical resection, when anatomically possible, is followed by a one month break and then chemoradiation and ionizing radiation (IR) induces DNA doublestrand breaks through direct high-energy damage to the sugar backbone of DNA, but also through free radicals generated in cells, which accounts for $60-70 \%$ of DNA lesions, exerting its genotoxic and ultimately cytotoxic effect $(3,4)$. However, glioblastoma is intrinsically resistant to IR (5-13), and therefore ionizing radiation therapy yields only marginal improvements in patient survival $(14,15)$, with a nearly $80 \%$ rate of recurrence within the high dose radiation field $(16,17)$. The nature of the resistance is unclear, but the general consensus is that it is related at least in part to increased glioblastoma tolerance to reactive oxygen species $(11,12,18-20)$ and enhanced GTP metabolism (21-23).

Stupp and colleagues described a therapeutic approach that remains the standard of care therapy for glioblastoma. The "Stupp protocol," as it is commonly 
called, uses IR plus concomitant and adjuvant temozolomide (TMZ, Temodar) (15). The addition of temozolomide, DNA-damage based treatment through alkylating guanine and adenine bases of DNA, to radiation, improves median survival by approximately an additional 6 months (15). It was noted that MGMT methylated glioblastomas fared better in terms of survival than MGMT unmethylated glioblastomas. These studies indicate that the efficacy of IR can be significantly improved by combination therapy. These results also point to the importance of mechanistic understanding of radioresistance and the identification of a new pathway that more effectively increases the efficacy of radiation on the tumor but not normal tissue.

Recently, Stupp and colleagues described the use of tumor treating fields (TTFs) with the standard of care therapy in glioblastoma (IR plus TMZ) in a randomized open-label trial of 695 glioblastoma patients, reporting that median progression-free survival was 6.7 months in the TTF plus standard of care group versus 4 months in the standard of care group alone (21). Phase 3 clinical trials of bevacizumab (Avastin) in glioblastoma showed no survival advantage; however, in glioblastoma patients with steroid dependence, bevacizumab can be used as a steroid-sparing agent (22). After the significant investment of resources into the upfront clinical trial setting of glioblastoma with immunotherapies, neoadjuvant anti-PD-1 therapy provided a promising survival benefit in recurrent glioblastoma (23), however, the Phase III study was negative in terms of survival benefit. Many vaccine trials are on the horizon for glioblastomas, and these are promising for patients who fulfill the selection criteria for these studies. However, many glioblastoma patients do not qualify for vaccine studies due to the location of the tumor, the immunogenicity of their tumor, and their performance status $(24,25)$. A critical challenge has been to develop new ways for accurate and rapid prediction of an individual patient's susceptibility to treatment.

Identifying a number of molecular genetic (for example, p53, NF1, PTEN, PDGFR) and signaling pathways (for example, RAS/ERK, PI3K/AKT pathways) involved in cancer development has led to several targeted agents being investigated in clinical evaluation for glioblastoma (26-28). To test the effects of the identified pathway and drug, preclinical mouse models have proven to be an invaluable tool, but unfortunately, none of these targets have been translatable in the clinical arena. Glioblastoma mouse models allow one to investigate basic mechanisms-enabling precise examination of numerous aspects, including cellular origins, regional differences in microenvironments, and the function of specific genetic events. However, a major challenge has been to develop preclinical mouse models that recapitulate the human glioblastoma nature in a time and cost-effective fashion. Furthermore, gliomas encompass a diverse set of tumors that can differ in location, age of onset, mutation status, and histopathological features making this an extraordinary challenge.

So, how can we change the prognosis of a patient with a glioblastoma? What target(s) should we be investigating as possible therapeutic vulnerabilities? How can we overcome the various barriers to generate and use glioblastoma mouse models? This chapter will review new advances in radiation therapeutics, molecular analysis of glioblastomas, and new animal models that will address these questions. 


\section{THE CURRENT STATE OF MOLECULAR ANALYSIS AND RELEVANCE IN GLIOBLASTOMA TREATMENT OPTIONS}

When a glioblastoma tumor is resected surgically, the tumor cells can be an invaluable source of information that can (and should) be utilized to guide patient treatment (personalized medicine). The tumor cells could potentially be used in a number of platforms, including RNA expression analysis (29-36), protein characterization (37-41), and metabolic profiling (37-39) (Figure 1). Once targets are identified, appropriate models can be generated to test treatment strategies, including 3D platforms such as ex vivo Organ-on-Chip (40, 41), in vitro cell culture methods including spheroids and organoids, and of course mouse models, transgenic and/or intracranial xenograft $(37,42-44)$ as well as large animals (for example, canine) $(45,46)$. Below we detail a newer emerging mouse model approach. Here we detail analysis of RNA sequencing to identify therapeutic targets and RNA-based therapeutics to target the mRNA or non-coding RNA.

\section{Analysis of mRNA in glioblastoma}

The genomic analyses of the tumor tissue or whole cells significantly increased our understanding of GBM in patients. For example, several whole-genome DNA sequencing studies reported (47) resulted in subtyping of glioblastomas based on their molecular phenotype. The IDH1/2-mutant GBMs are distinct categories, for example, mutations in the EGFR, PDGFRA, NF1 genes; mutations in hTERT promoter; epigenetic changes such as altered methylation of the MGMT gene promoter $(48,49)$. While advanced genetic analysis has improved the prediction of glioma outcomes and treatment approaches (50-52), unpredictable significant inter-individual variation in therapeutic efficacy is often seen in the treatment of glioblastoma patients. Apart from the tumor itself, glioblastomas are heterogeneous, composed of diverse cells, such as immune cells and stromal cells $(53,54)$.

Two of the significant challenges are understanding the pathogenesis of glioblastoma and predicting more accurately patient sensitivity to a selected treatment(s). The analysis of individual parts of the genome and the transcribed RNA provides the potential for a more comprehensive understanding of the pathogenesis of glioma and possible treatments tailored to individual patients. The recent development of single-cell RNA-sequencing (scRNA-seq) has enabled functional analysis of individual glioblastoma cells. The differential transcriptional landscape between individual cells can have profound functional consequences, for example, cellular, molecular, genetic, epigenetic, and metabolic heterogeneity. These heterogeneities affect the course of glioblastoma development and therapeutic responses, challenging personalized medicine. Historically, single-cell transcriptional analysis is started by single-cell quantitative PCR (qPCR). Rapid progress in the development of sequencing and single-cell isolation technologies in recent years has enabled RNA sequencing at single-cell levels (55). The scRNAseq technology can uncover highly complex as well as rare cell populations. Also, the time-resolved scRNA-seq analysis can clarify the regulatory relationship between gene expression in responses to stimuli (for example, therapeutic treatment) and track the trajectories of cell lineages. 


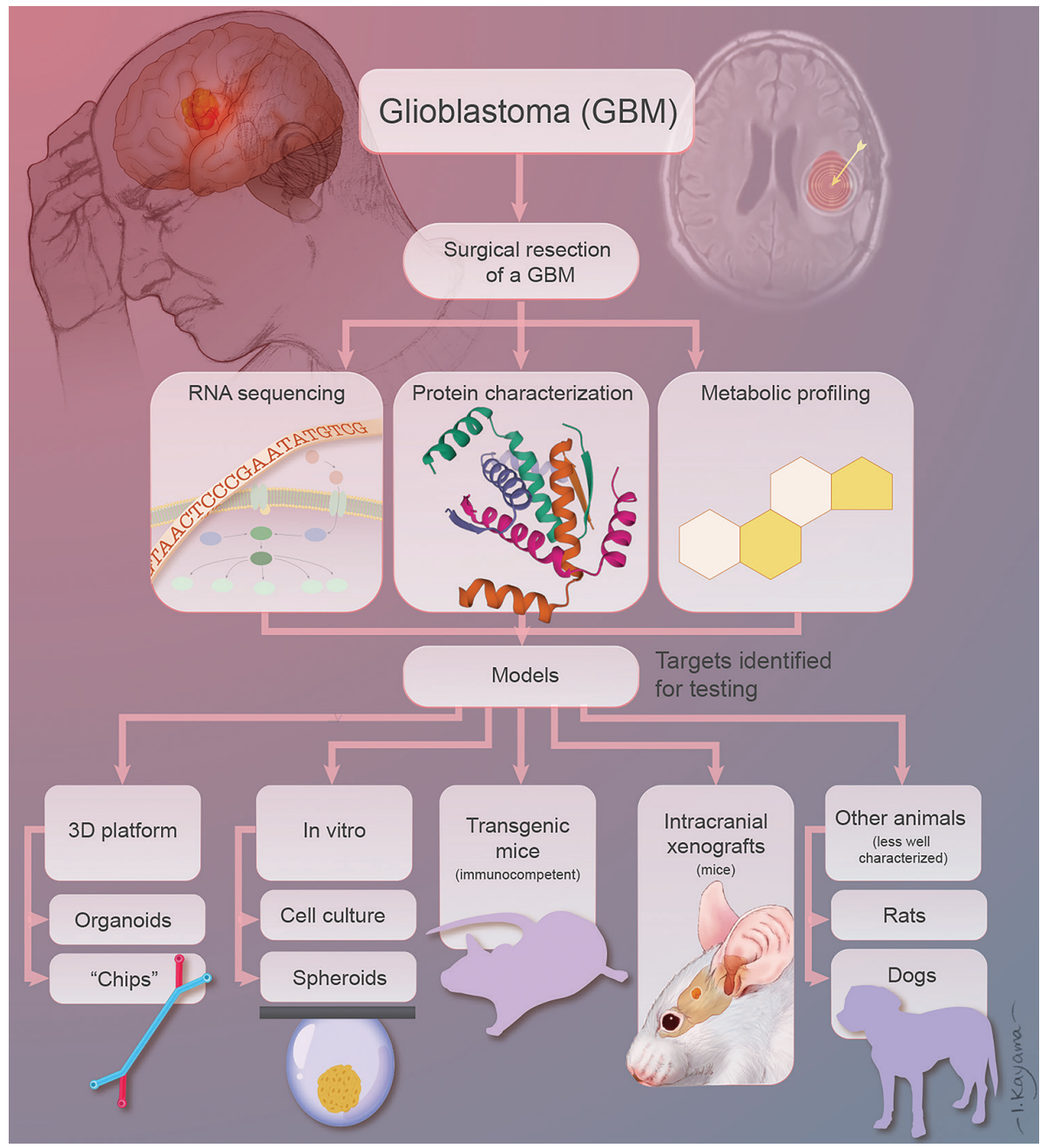

Figure 1. A flow diagram of a personalized medicine approach of a patient's glioblastoma tumor. This diagram shows how a patient with a glioblastoma experiencing headaches, has imaging that localizes a tumor. When the tumor is resected surgically, it could potentially be used in a number of platforms, including, RNA sequencing, protein characterization and metabolic profiling. Once targets are identified the appropriate models can be generated as shown in the flow diagram, including, 3D platforms, in vitro cell culture methods, transgenic mice, intracranial xenografts and large animal studies.

Suva and colleagues (56) utilized scRNA-seq analysis of 28 glioblastomas and bulk expression analysis of 401 specimens from the TCGA, combined with functional approaches and single-cell lineage tracing to create a cohesive model for the genetic heterogeneity and cellular states in glioblastomas. They found that malignant cell niches in glioblastomas are influenced by copy number amplifications of CDK4, EGFR, and PDGFR loci and mutations in NF1. Couturier et al. (57), utilizing scRNA-seq analysis of 53,586 adult glioblastoma cells and 22,637 normal 
human fetal brain cells, reported a conserved neural tri-lineage hierarchy centered around glial progenitor-like cells. They reported that within this progenitor population, most cancer cells are centered around said glial progenitor-like cells and that the course of glioblastoma development is along with the conserved neurodevelopmental gene programs, which possesses a rapidly dividing progenitor population. Their scRNA-seq analysis revealed new insight on primary glioblastomas and created a hierarchical map to identify therapeutic targets specific to progenitor cancer stem cells.

\section{Analysis of non-coding RNA in glioblastoma}

While there is an emphasis placed on the study of the expression of proteincoding genes (mRNA transcripts), it is essential not to overlook the potential contribution of non-coding genes as well as promoter and enhancer elements. Functional studies in disparate systemic cancers have shown that long non-coding RNAs and miRNAs can promote pathogenesis. Integration analyses of long noncoding RNAs and competing endogenous RNA networks in glioblastomas are much rarer. These networks may be the key to understanding the pathogenesis of glioblastomas, as well as predicting the therapeutic efficacy of the selected treatment, thus representing an untapped therapeutic potential (58).

\section{RNA-based therapies and their delivery to a glioblastoma}

We suspect that many potential targets will not have targeted drugs available. Thus, we should keep open the possibility of targeting transcripts and/or noncoding RNAs with RNA-based therapeutics (such as siRNAs, ADARs, gRNA for a CRISPR-Cas9 approach) and the development of technologies that can aid their delivery to a glioblastoma. On this front, an emerging clinical technology that may significantly impact glioblastoma treatment is the application of focused ultrasound combined with microbubbles to facilitate the delivery of RNA-based therapeutics as well as large drugs and monoclonal antibodies into glioblastomas $(59,60)$. Importantly, this non-invasive technology can overcome the bloodbrain/blood-tumor barriers for delivery of RNA-based therapeutics and the nanoparticles used to aid their delivery, but many agents that might be effective in vitro but because of their size/mass cannot cross the blood-brain/blood-tumor barriers, for example, earlier generations of EGFR tyrosine kinase inhibitors and newer agents such as monoclonal antibodies and vaccines $(61,62)$.

\section{EMERGING MOUSE MODELS OF PEDIATRIC AND ADULT HIGH-GRADE GLIOMA AND GLIOBLASTOMA}

Developing animal models that faithfully recapitulate the features of human gliomas is essential for conducting accurate preclinical studies that facilitate the development of novel drugs and therapeutic strategies that can be translated into the clinic. While the establishment of patient-derived cell lines and xenograft models has dramatically advanced our understanding of this deadly disease, limitations 
for patient-derived models include the inability to establish consistent cultures from tumors, the presence of numerous and complex genetic events, and the use of immunodeficient hosts, which limits their use for immunotherapy studies (63). Traditionally, immunocompetent glioblastoma mouse models have been generated using the knock-out and transgenic mouse approach. However, the knockout and transgenic-mouse approach often require crossing with other mutant mice (for example, Trp53-KO, Pten-KO), which takes a number of years and costs to maintain the colonies (63). The replication-competent ALV splice acceptor (RCAS)/tv-a glioblastoma system developed by Holland's group overcomes this issue $(64,65)$. The engineered RCAS virus carrying the gene of interest (for example, EGFR, shRNA for PTEN, sgRNA for p53) can be delivered to the specific cells (for example, glia) that is engineered to express its receptor $t-v a(64,65)$. While the RCAS/t-va system is a revolutionized technology, potential limitations are that the RCAS virus vector typically allows the small size of the insert $(3 \mathrm{~kb})$, and it requires $t$-va expressing transgenic mouse; thus, the system is basically incompatible with the existing mutant mouse. In this section, we highlight in utero electroporation (IUE) based glioma mouse models, which have been developed for both adult and pediatric gliomas (Figure 2).

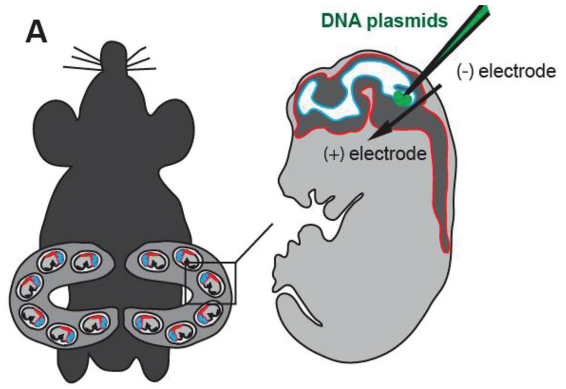

B

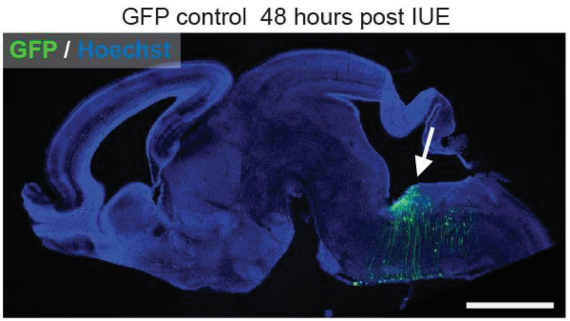

C

D
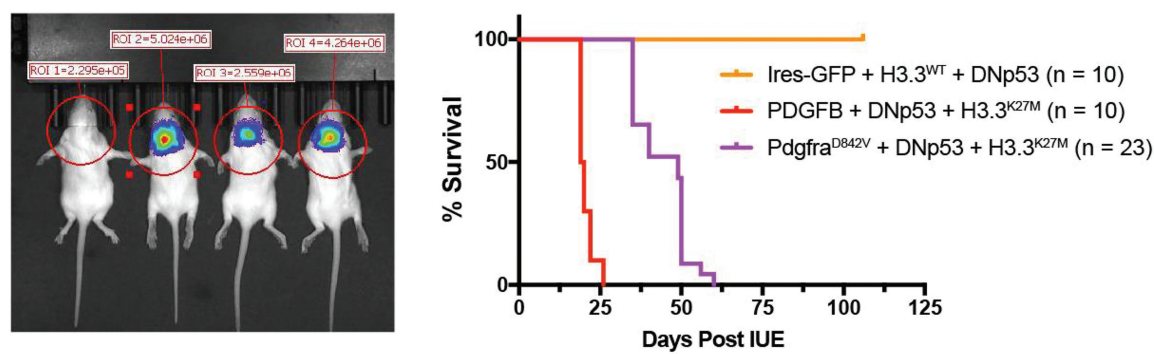

Figure 2. In utero electroporation-based models of DIPG. A. Schematic cartoon of the IUE procedure targeting the developing embryonic brainstem. B. Representative image of GFP-positive transfected brainstem neural stem and progenitor cells 48 hours post brainstem IUE in a sagittal brain section. C. Example of luciferase positive and negative IUE pups at two weeks postnatal. D. Survival curve for different oncogenic IUE combinations depicting different rates of tumorigenesis. 


\section{In utero electroporation-based glioma mouse models}

To address some of the drawbacks of traditional genetic mouse models, including high cost and lengthy development times, a number of groups have utilized IUE as a flexible and rapid platform to develop glioma mouse models. IUE of the developing brain was first described by Tabata and Nakajima in 2001 (66) and has traditionally been used to label and track cellular migration or the impact of transient genetic manipulations on cellular proliferation and differentiation (67). More recently, a number of laboratories have paired this technique with advances in DNA editing technologies to develop new brain tumor mouse models (68-70). First, the application of CRISPR-Cas9 technology to directly edit the mouse genome has been successfully used to generate adult glioma mouse models (68). By transfecting guide RNAs (gRNA) targeting known glioma mutations, such as Nf1, Trp53, and Pten, along with the Cas9 protein, Zuckermann et al. first described this efficient method to create triple loss-of-function mutant adult glioma mouse models using IUE (68). They validated the effectiveness of CRISPR-Cas9 gRNAs at generating frameshift INDELs within targeted genes; successfully targeted IUE offspring developed fully penetrant gliomas. This triple-CRISPR model has been used by a number of other groups, providing a platform to screen genetic variants $(71,72)$ and study functional interactions between tumor cells and the microenvironment (73).

The ability to restrict transfection to specific brain areas with IUE also provides an opportunity to model regionally distinct glioma subtypes, such as diffuse intrinsic pontine glioma (DIPG), a highly lethal pediatric glioma that arises in the brainstem (74-76). Compared to adult gliomas, DIPGs harbor unique genetic alterations, including histone $\mathrm{K} 27 \mathrm{M}$ mutations that are found in approximately $80 \%$ of cases (77). The use of traditional genetic mouse models to model histone mutations did not result in glioma formation, yet the introduction of histone K27M mutations, paired with Tp53 loss-of-function and Pdgfra expression by cortical or brainstem targeted IUE produced fully penetrant gliomas (78). Additional IUE based DIPG mouse models generated by Patel et al. revealed a range of latencies, histopathologies, and gene expression changes induced by specific combinations of mutations used to model DIPG (70). In this study, they noted the importance of histone $\mathrm{K} 27 \mathrm{M}$ mutations in accelerating glioma formation in the presence of Pdgfra ${ }^{\mathrm{D} 842 \mathrm{~V}}+\mathrm{DN}-\mathrm{p} 53$ (dominant-negative p53) and its requirement for tumor formation in WT-Pdgfra + DN-p53 backgrounds. Besides hastening glioma development, H3 K27M mutations drove epigenetic and transcriptional changes that mirror those identified in K27M mutant DIPG patient samples, including loss of H3K27me3 (79) and decreased CDKN2A (p16) expression, respectively $(80,81)$.

Developing animal models that recapitulate the features of human gliomas is essential for conducting accurate preclinical studies to improve the prediction of drug penetration and efficacy, as well as radiation efficacy. Utilizing the IUE platform to generate regionally and genetically distinct glioma mouse model in a rapid and flexible manner provides exciting new research possibilities. This includes investigating recently identified mutations discovered by next-generation sequencing studies of patient samples or targeting DNA transfection to specific cell-types to test the impact of cellular origin on tumorigenesis. In addition, the immune competency status of these IUE glioma mouse models also provides an in vivo 
system to examine immune-modulatory and immunotherapy strategies. Our recent studies have shown that an IUE-based high-grade glioma is a powerful approach, revealing that the efficacy of dasatinib treatment of PdgfraD842V + DN-p53 high-grade glioma is enhanced with everolimus (82). The results suggest a promising route for improving targeted therapy for high-grade glioma patient with the driver mutation of PDGF $\alpha$ and $\mathrm{p} 53$.

\section{PROTON BEAM THERAPY}

Theoretically, higher doses of radiation increase the anti-glioma effect. However, an increase in dose is generally associated with an increased risk of radiation necrosis and toxicity to normal surrounding tissue (83). Various technological advances have led to improved delivery systems in radiotherapy. Contact radiotherapy via radioactive sources and superficial energies was superseded by 2-dimensional radiotherapy with the ability to generate increasing energies of electrons and generated photons. This advanced with the advent of computed tomography to 3-dimensional treatment. Intensity-modulated radiotherapy, improving conformality, and advanced planning techniques were made possible by computer technology improvements (84). In addition, significant advances in the treatment of malignancies were made in the ability to use various particles. In this section, we focus on proton beam therapy (PBT), one of the most precise and advanced forms of radiation therapy available in the world today.

\section{Stereotactic radiosurgery}

Stereotactic radiosurgery may also be considered in the upfront or the more commonly recurrent setting for glioma treatment. This is an ablative therapy of very high radiotherapy doses administered over traditionally one, but up to five fractions. In the upfront setting, consideration is primarily focused on decreasing the number of fractions received, typically spanning six weeks for 30 total treatments (85). For treatment at the time of relapse, both fractionated and stereotactic radiotherapy may be considered. As at the time of presentation, fractionation allows for the treatment of larger volumes commonly associated with these infiltrative tumors. Yet, radiosurgery may be appealing for both conveniences as well as it is biologically appealing to overcome some of the radioresistant mechanism of gliomas (86). Future directions within radiotherapy in glioma treatment will continue to improve upon advanced planning and delivery systems. In addition, combinatorial therapies maximizing efficacy and minimizing toxicity continue to be investigated.

\section{Proton beam therapy leads to fewer side effects and complications}

Following photon radiation (ionizing radiation), proton radiation has become the predominant modality used in the treatment of brain tumors. Due to the relatively low mass and lack of charge, photons slowly lose energy along a path length extending through the entirety of the patient. Conversely, due to high relative mass and associated charge, protons have a dose deposition concentrated near the 


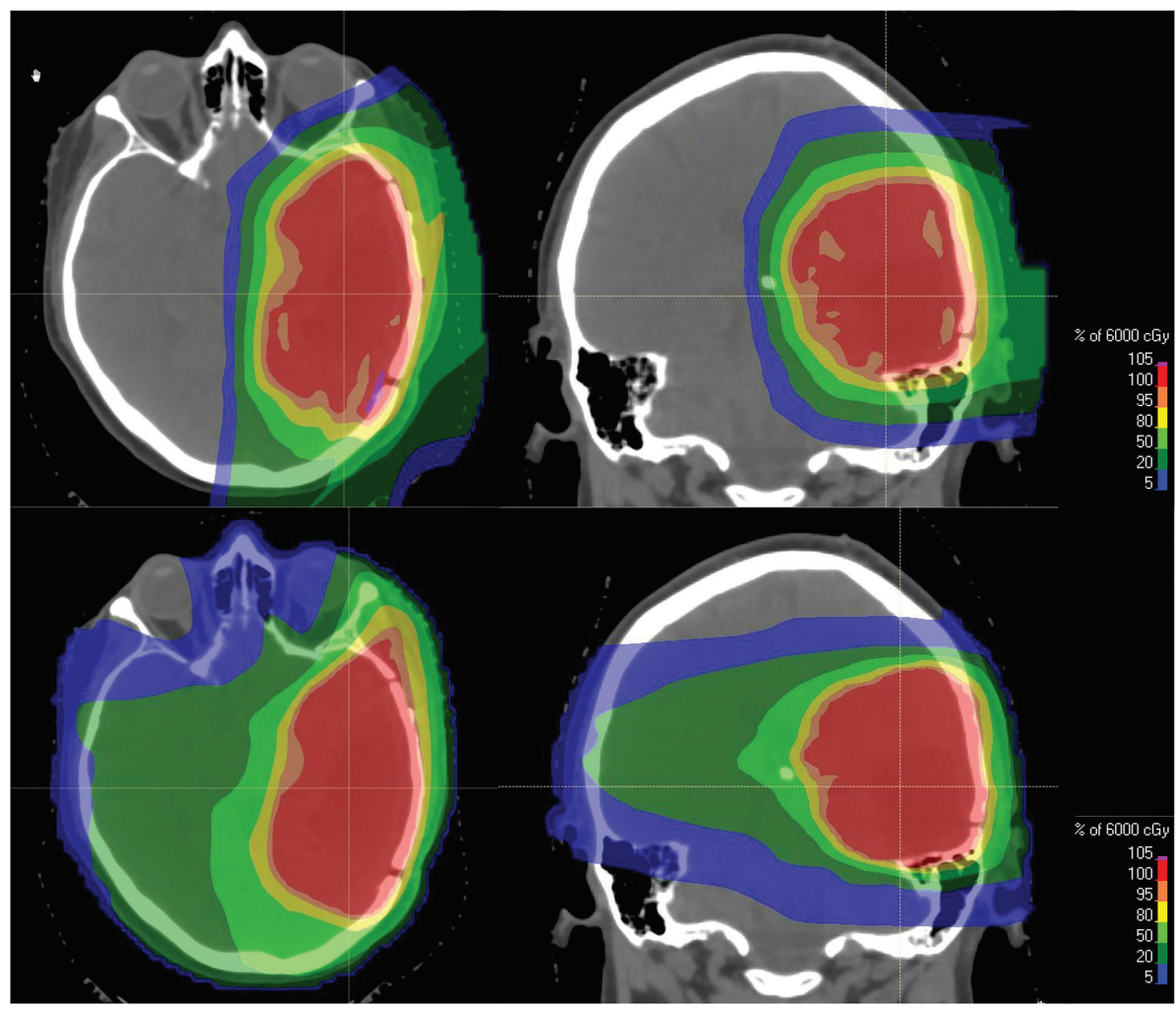

Figure 3. Comparative dosimetry for left temporal glioma radiotherapy treatment with protons (upper) and photons (lower). Prescription is for 60 Cobalt Gray Equivalent in 30 fractions. Noted decrease in radiotherapy exposure to the eyes, optic apparatus, brainstem, and contralateral structures.

end of their range, allowing the bulk of radiation dose deposited in a highly confined area, termed the "Bragg peak" (Figure 3). The Bragg peak is the characteristic dose deposition profile of a proton with nearly all energy loss just prior to its end of the range (87). This allows for a decrease in radiation exposure downstream from the target tissue, decreasing the side effects and complications of radiotherapy.

The superiority in the conformality and accuracy of proton beam therapy is a critical advantage for the treatment of brain tumors, circumventing the side effects and potentially allowing for an increase in the administered dose to the tumor. Proton beam radiotherapy has the capability to reduce the dose to non-tumor tissue in virtually all tumor locations within the brain $(88,89)$. The magnitude and clinical benefit of these reductions are variable and require clinical determination by the radiation oncologist. In the pediatric setting where radiation exposure to developing tissue is of the highest concern, studies have shown detriment to cognition and neuroendocrine function from radiotherapy exposures (90). Dosimetric studies have confirmed a decrease in radiation exposure to 
structures associated with memory and cognition utilizing proton radiotherapy (91). Early studies reporting neurocognitive outcomes in pediatric patients undergoing proton beam therapy correlate these dosimetric changes to favorable outcomes compared to similar photon historical cohorts $(92,93)$.

The use of proton radiotherapy in adult brain tumors was constrained by the availability of these machines and was, therefore, initially reserved predominantly for tumors considered radioresistant, thus requiring higher doses. Representative tumors include skull base chordoma and chondrosarcoma, and uveal melanoma, as well as some benign processes (94). With the expanding number of facilities increasing access to proton radiotherapy, indications for proton radiotherapy have similarly increased. Brain tumors of favorable prognosis in the adult population are now considered for proton radiotherapy to minimize long-term toxicity. In addition, proton radiotherapy may be considered in cases of reirradiation where critical structures may have been previously exposed to doses at the threshold of significant risk for toxicity or to minimize the quality of life detriment from large cumulative exposures (95).

\section{Proton beam therapy center with a capability for basic research}

Proton beam therapy has been used clinically for over 50 years. However, proton beam therapy was only available for a few populations of patients due to its cost and limited availability. Thanks to significant advances in technology, there are now 31 proton therapy centers operative in the USA today. As the numbers of proton centers increase, there has been more consideration for the basic research requirements that would expand the potential application of proton therapy and improve its efficacy. Towards this end, Cincinnati Children's Hospital invested $\$ 120$ million and launched one of the most advanced proton research and cancer treatment centers in the world in 2016. The facility includes several tracks for basic biological research to investigate the cellular responses of tumors to proton radiation and for translational research to develop and refine other treatments that can augment proton therapy. While the majority of current research is undertaken by cooperation among the local entities (Cincinnati Children's Hospital Medical Center and the University of Cincinnati), collaborations across countries and continents are widely open in many directions and ongoing.

\section{CONCLUSION}

The current standard of care for glioblastoma is surgical resection followed by radiation combined with temozolomide. Adjuvant therapy is vital because glioblastoma grows invasively in the surrounding brain tissue and, almost invariably, is rarely completely resectable. Patient response to treatment is often unpredictable and can differ significantly from experimental results in mice. A critical challenge is to develop new ways for accurate and rapid prediction of an individual patient's susceptibility to treatment. In this chapter, we have discussed three approaches to achieve this goal: (i) new genomic and RNA molecular analysis, (ii) an enhanced glioblastoma mouse model using IUE technology, and (iii) improved radiotherapy using proton therapy. 
Integrating sophisticated pathway analysis with mouse models that recapitulate the human disease coupled with the ability to perform basic studies on the mechanisms of photon therapy has a high potential to overcome the current challenges in GBM therapy. While this needs an establishment of a framework to proceed with a sample obtained from a patient and link the result of the approaches, once established, we expect that the combination of these three approached with the current standard of care will enable choosing the best treatment and markedly improve glioblastoma outcomes.

Acknowledgment: We thank Ms. Ikumi Kayama for providing the illustration of the Figurel, and Dr. Eric Smith for the critical proofreading. This work has been supported by the MTP UC-Brain Tumor Center grant, Ohio Cancer Research, B*Cured, R21NS100077, and R01NS089815 (A.T.S.), the Fund for the Promotion of Joint International Research (Fostering Joint International Research (A)) from the JSPS, 18KK0455 (Y.H.), and the Harold C. Schott Foundation, and the Pam and Tom Mischell Funds (S.S.). This work was supported in part by research funds from the Yamagata prefectural government and the City of Tsuruoka.

Conflict of interest: The authors declare no potential conflicts of interest with respect to research, authorship, and/or publication of this chapter.

Copyright and Permission Statement: The authors confirm that the materials included in this chapter do not violate copyright laws. Where relevant, appropriate permissions have been obtained from the original copyright holder(s), and all original sources have been appropriately acknowledged or referenced.

\section{REFERENCES}

1. Louis DN, Perry A, Reifenberger G, Deimling von A, Figarella-Branger D, Cavenee WK, et al. The 2016 World Health Organization Classification of Tumors of the Central Nervous System: a summary. Acta Neuropathol. Springer Berlin Heidelberg; 2016;131(6):803-20. https://doi.org/10.1007/ s00401-016-1545-1

2. RossJL, Cooper LAD, KongJ, Gutman D, Williams M, Tucker-Burden C, et al. 5-Aminolevulinic Acid Guided Sampling of Glioblastoma Microenvironments Identifies Pro-Survival Signaling at Infiltrative Margins. Sci Rep. Nature Publishing Group; 2017;7(1):15593-11. https://doi.org/10.1038/s41598-017-15849-w

3. Ward JF. Biochemistry of DNA lesions. Radiat Res Suppl. 1985;8:S103-11. https://doi.org/10.2307 13583517

4. Powell S, McMillan TJ. DNA damage and repair following treatment with ionizing radiation. Radiother Oncol. 1990;19(2):95-108. https://doi.org/10.1016/0167-8140(90)90123-E

5. Laperriere N, Zuraw L, Cairncross G, Cancer Care Ontario Practice Guidelines Initiative NeuroOncology Disease Site Group. Radiotherapy for newly diagnosed malignant glioma in adults: a systematic review. Radiother Oncol. 2002;64(3):259-73. https://doi.org/10.1016/S0167-8140(02)00078-6

6. Grossman SA, Batara JF. Current management of glioblastoma multiforme. Semin Oncol. 2004;31(5): 635-44. https://doi.org/10.1053/j.seminoncol.2004.07.005

7. Furnari FB, Fenton T, Bachoo RM, Mukasa A, Stommel JM, Stegh A, et al. Malignant astrocytic glioma: genetics, biology, and paths to treatment. Genes \& Dev. Cold Spring Harbor Lab; 2007;21(21):2683-710. https://doi.org/10.1101/gad.1596707

8. Chalmers AJ. Radioresistant glioma stem cells--therapeutic obstacle or promising target? DNA Repair. 2007;6(9):1391-4. https://doi.org/10.1016/j.dnarep.2007.03.019 
9. Wang J, Wakeman TP, Lathia JD, Hjelmeland AB, Wang X-F, White RR, et al. Notch promotes radioresistance of glioma stem cells. STEM CELLS. John Wiley \& Sons, Ltd; 2010;28(1):17-28. https://doi. org/10.1002/stem.261

10. Hatanpaa KJ, Burma S, Zhao D, Habib AA. Epidermal growth factor receptor in glioma: signal transduction, neuropathology, imaging, and radioresistance. Neoplasia. 2010;12(9):675-84. https://doi. org/10.1593/neo.10688

11. Bao S, Wu Q, McLendon RE, Hao Y, Shi Q, Hjelmeland AB, et al. Glioma stem cells promote radioresistance by preferential activation of the DNA damage response. Nature. 2006;444(7120):756-60. https://doi.org/10.1038/nature05236

12. Matlaf L. Reactive oxygen species-mediated therapeutic response and resistance in glioblastoma. Cell Death Dis. 2015;6(1):e1601-1. https://doi.org/10.1038/cddis.2014.566

13. Han X, Xue X, Zhou H, Zhang G. A molecular view of the radioresistance of gliomas. Oncotarget. Impact Journals; 2017;8(59):100931-41. https://doi.org/10.18632/oncotarget.21753

14. Stupp R, Hegi ME, Mason WP, van den Bent MJ, Taphoorn MJ, Janzer RC, et al. Effects of radiotherapy with concomitant and adjuvant temozolomide versus radiotherapy alone on survival in glioblastoma in a randomised phase III study: 5-year analysis of the EORTC-NCIC trial. Lancet Oncol. Elsevier; 2009;10(5):459-66. https://doi.org/10.1016/S1470-2045(09)70025-7

15. Stupp R, Mason WP, Van Den Bent MJ, Weller M, Fisher B, Taphoorn MJB, et al. Radiotherapy plus concomitant and adjuvant temozolomide for glioblastoma. N Engl J Med. 2005;352(10):987-96. https://doi.org/10.1056/NEJMoa043330

16. Brandes AA, Tosoni A, Franceschi E, Sotti G, Frezza G, Amistà P, et al. Recurrence pattern after temozolomide concomitant with and adjuvant to radiotherapy in newly diagnosed patients with glioblastoma: correlation With MGMT promoter methylation status. J Clin Oncol. 2009;27(8):1275-9. https://doi.org/10.1200/JCO.2008.19.4969

17. Gebhardt BJ, Dobelbower MC, Ennis WH, Bag AK, Markert JM, Fiveash JB. Patterns of failure for glioblastoma multiforme following limited-margin radiation and concurrent temozolomide. Radiat Oncol. 2014;9(1):130. https://doi.org/10.1186/1748-717X-9-130

18. Rinaldi M, Caffo M, Minutoli L, Marini H, Abbritti RV, Squadrito F, et al. ROS and Brain Gliomas: An Overview of Potential and Innovative Therapeutic Strategies. IJMS. Multidisciplinary Digital Publishing Institute; 2016;17(6):984. https://doi.org/10.3390/ijms17060984

19. Salazar-Ramiro A, Ramírez-Ortega D, Pérez de la Cruz V, Hérnandez-Pedro NY, González-Esquivel DF, Sotelo J, et al. Role of Redox Status in Development of Glioblastoma. Front Immunol. 2016;7(2): 157-15. https://doi.org/10.3389/fimmu.2016.00156

20. Polewski MD, Reveron-Thornton RF, Cherryholmes GA, Marinov GK, Aboody KS. SLC7A11 Overexpression in Glioblastoma Is Associated with Increased Cancer Stem Cell-Like Properties. Stem Cells Dev. 201;26(17):1236-46. https://doi.org/10.1089/scd.2017.0123

21. Stupp R, Taillibert S, Kanner A, Read W, Steinberg D, Lhermitte B, et al. Effect of Tumor-Treating Fields Plus Maintenance Temozolomide vs Maintenance Temozolomide Alone on Survival in Patients With Glioblastoma: A Randomized Clinical Trial. JAMA. 2017;318(23):2306-16. https://doi.org/10.1001/ jama.2017.18718

22. Kim MM, Umemura Y, Leung D. Bevacizumab and Glioblastoma: Past, Present, and Future Directions. Cancer J. 2018;24(4):180-6. https://doi.org/10.1097/PPO.0000000000000326

23. Cloughesy TF, Mochizuki AY, Orpilla JR, Hugo W, Lee AH, Davidson TB, et al. Neoadjuvant anti-PD-1 immunotherapy promotes a survival benefit with intratumoral and systemic immune responses in recurrent glioblastoma. Nat. Med. Nature Publishing Group; 2019;25(3):477-86.

24. Sampson JH, Gunn MD, Fecci PE, Ashley DM. Brain immunology and immunotherapy in brain tumours. Nat Rev Cancer. Nature Publishing Group; 2020;20(1):12-25. https://doi.org/10.1038/ s41568-019-0224-7

25. Winograd EK, Ciesielski MJ, Fenstermaker RA. Novel vaccines for glioblastoma: clinical update and perspective. Immunotherapy. Future Medicine Ltd London, UK; 2016;8(11):1293-308. https://doi. org/10.2217/imt-2016-0059

26. Wang H, Xu T, Jiang Y, Xu H, Yan Y, Fu D, et al. The challenges and the promise of molecular targeted therapy in malignant gliomas. Neoplasia. 2015;17(3):239-55. https://doi.org/10.1016/j. neo.2015.02.002 
27. Bastien JIL, McNeill KA, Fine HA. Molecular characterizations of glioblastoma, targeted therapy, and clinical results to date. Cancer. 2015;121(4):502-16. https://doi.org/10.1002/cncr.28968

28. Pearson JRD, Regad T. Targeting cellular pathways in glioblastoma multiforme. Signal Transduct Target Ther. 2017;2(1):17040. https://doi.org/10.1038/sigtrans.2017.40

29. Shai R, Shi T, Kremen TJ, Horvath S, Liau LM, Cloughesy TF, et al. Gene expression profiling identifies molecular subtypes of gliomas. Oncogene. Nature Publishing Group; 2003;22(31):4918-23. https:// doi.org/10.1038/sj.onc. 1206753

30. Liang Y, Diehn M, Watson N, Bollen AW, Aldape KD, Nicholas MK, et al. Gene expression profiling reveals molecularly and clinically distinct subtypes of glioblastoma multiforme. Proc Natl Acad Sci USA. National Academy of Sciences; 2005;102(16):5814-9. https://doi.org/10.1073/ pnas.0402870102

31. Tso C-L, Freije WA, Day A, Chen Z, Merriman B, Perlina A, et al. Distinct transcription profiles of primary and secondary glioblastoma subgroups. Cancer Research. American Association for Cancer Res. 2006;66(1):159-67. https://doi.org/10.1158/0008-5472.CAN-05-0077

32. Sun J, Gong X, Purow B, Zhao Z. Uncovering MicroRNA and Transcription Factor Mediated Regulatory Networks in Glioblastoma. Goryachev AB, editor. PLoS Comp Biol. 2012;8(7):e1002488. https://doi. org/10.1371/journal.pcbi.1002488

33. Patel AP, Tirosh I, Trombetta JJ, Shalek AK, Gillespie SM, Wakimoto H, et al. Single-cell RNA-seq highlights intratumoral heterogeneity in primary glioblastoma. Science. 2014;344(6190):1396-401. https://doi.org/10.1126/science.1254257

34. Ceccarelli M, Barthel FP, Malta TM, Sabedot TS, Salama SR, Murray BA, et al. Molecular Profiling Reveals Biologically Discrete Subsets and Pathways of Progression in Diffuse Glioma. Cell. 2016;164(3):550-63.

35. Darmanis S, Sloan SA, Croote D, Mignardi M, Chernikova S, Samghababi P, et al. Single-Cell RNASeq Analysis of Infiltrating Neoplastic Cells at the Migrating Front of Human Glioblastoma. Cell Rep. 2017;21(5):1399-410. https://doi.org/10.1016/j.celrep.2017.10.030

36. Mack SC, Singh I, Wang X, Hirsch R, Wu Q, Villagomez R, et al. Chromatin landscapes reveal developmentally encoded transcriptional states that define human glioblastoma. J Exp Med. 2019;216(5):1071-90. https://doi.org/10.1084/jem.20190196

37. Marin-Valencia I, Yang C, Mashimo T, Cho S, Baek H, Yang X-L, et al. Analysis of Tumor Metabolism Reveals Mitochondrial Glucose Oxidation in Genetically Diverse Human Glioblastomas in the Mouse Brain In\&nbsp;Vivo. Cell Metab. Elsevier Inc; 2012;15(6):827-37. https://doi.org/10.1016/j. cmet.2012.05.001

38. Heiland DH, Wörner J, Gerrit Haaker J, Delev D, Pompe N, Mercas B, et al. The integrative metabolomic-transcriptomic landscape of glioblastome multiforme. Oncotarget. 2017;8(30):49178-90. https://doi.org/10.18632/oncotarget.16544

39. Arias-Ramos N, Ferrer-Font L, Lope-Piedrafita S, Mocioiu V, Julià-Sapé M, Pumarola M, et al. Metabolomics of Therapy Response in Preclinical Glioblastoma: A Multi-Slice MRSI-Based Volumetric Analysis for Noninvasive Assessment of Temozolomide Treatment. Metabolites. 2017;7(2):20. https:// doi.org/10.3390/metabo7020020

40. Jacob F, Salinas RD, Zhang DY, Nguyen PTT, Schnoll JG, Wong SZH, et al. A Patient-Derived Glioblastoma Organoid Model and Biobank Recapitulates Inter- and Intra-tumoral Heterogeneity. Cell. 2020;180(1):188-204.e22. https://doi.org/10.1016/j.cell.2019.11.036

41. Azzarelli R. Organoid Models of Glioblastoma to Study Brain Tumor Stem Cells. Front Cell Dev Biol. 2020;8:220. https://doi.org/10.3389/fcell.2020.00220

42. Marin-Valencia I, Cho SK, Rakheja D, Hatanpaa KJ, Kapur P, Mashimo T, et al. Glucose metabolism via the pentose phosphate pathway, glycolysis and Krebs cycle in an orthotopic mouse model of human brain tumors. NMR Biomed. 2012;25(10):1177-86. https://doi.org/10.1002/nbm.2787

43. Mashimo T, Pichumani K, Vemireddy V, Hatanpaa KJ, Singh DK, Sirasanagandla S, et al. Acetate Is a Bioenergetic Substrate for Human Glioblastoma and Brain Metastases. Cell. 2014;159(7):1603-14. https://doi.org/10.1016/j.cell.2014.11.025

44. Kofuji S, Hirayama A, Eberhardt AO, Kawaguchi R, Sugiura Y, Sampetrean O, et al. IMP dehydrogenase-2 drives aberrant nucleolar activity and promotes tumorigenesis in glioblastoma. Nat Cell Biol. Springer US; 2019;21(8):1-18. https://doi.org/10.1038/s41556-019-0363-9 
45. Candolfi M, Curtin JF, Nichols WS, Muhammad AG, King GD, Pluhar GE, et al. Intracranial glioblastoma models in preclinical neuro-oncology: neuropathological characterization and tumor progression. J Neurooncol. 2007;85(2):133-48. https://doi.org/10.1007/s11060-007-9400-9

46. De Vleeschouwer S, Rossmeisl J. Maximizing Local Access to Therapeutic Deliveries in Glioblastoma. Part V: Clinically Relevant Model for Testing New Therapeutic Approaches. Glioblastoma. Brisbane (AU): Codon Publications; 2017;405-25. https://doi.org/10.15586/codon.glioblastoma.2017.ch21

47. Aldape K, Zadeh G, Mansouri S, Reifenberger G, Deimling von A. Glioblastoma: pathology, molecular mechanisms and markers. Acta Neuropathol. Springer Berlin Heidelberg; 2015;129(6):829_48. https://doi.org/10.1007/s00401-015-1432-1

48. Verhaak RGW, Hoadley KA, Purdom E, Wang V, Qi Y, Wilkerson MD, et al. Integrated genomic analysis identifies clinically relevant subtypes of glioblastoma characterized by abnormalities in PDGFRA, IDH1, EGFR, and NF1. Cancer Cell. Elsevier Ltd; 2010;17(1):98-110.

49. Neftel C, Laffy J, Filbin MG, Hara T, Shore ME, Rahme GJ, et al. An Integrative Model of Cellular States, Plasticity, and Genetics for Glioblastoma. Cell. 2019;178(4):835-849.e21.

50. Brennan CW, McKenna A, Campos B, Noushmehr H, Salama SR, Zheng S, et al. The Somatic Genomic Landscape of Glioblastoma. Cell. Elsevier Inc; 2013;155(2):462-77.

51. Masui K, Mischel PS, Reifenberger G. Molecular classification of gliomas. Handb Clin Neurol. Elsevier; 2016;134:97-120. https://doi.org/10.1016/B978-0-12-802997-8.00006-2

52. Aquilanti E, Miller J, Santagata S, Cahill DP, Brastianos PK. Updates in prognostic markers for gliomas. Neuro Oncol. 2018;20(suppl_7):viil7-vii26. https://doi.org/10.1093/neuonc/noyl58

53. Vartanian A, Singh SK, Agnihotri S, Jalali S, Burrell K, Aldape KD, et al. GBM's multifaceted landscape: highlighting regional and microenvironmental heterogeneity. Neuro Oncol. 2014;16(9):1167-75. https://doi.org/10.1093/neuonc/nou035

54. Martinez-Lage M, Lynch TM, Bi Y, Cocito C, Way GP, Pal S, et al. Immune landscapes associated with different glioblastoma molecular subtypes. Acta Neuropathol Commun. 2019;7(1):203. https://doi. org/10.1186/s40478-019-0803-6

55. Kolodziejczyk AA, Kim JK, Svensson V, Marioni JC, Teichmann SA. The technology and biology of single-cell RNA sequencing. Mol Cell. 2015;58(4):610-20. https://doi.org/10.1016/j.molcel. 2015.04.005

56. Suvà ML, Rheinbay E, Gillespie SM, Patel AP, Wakimoto H, Rabkin SD, et al. Reconstructing and Reprogramming the Tumor-Propagating Potentialof Glioblastoma Stem-like Cells. Cell. Elsevier Inc; 2014;157(3):580-94. https://doi.org/10.1016/j.cell.2014.02.030

57. Couturier CP, Ayyadhury S, Le PU, Nadaf J, Monlong J, Riva G, et al. Single-cell RNA-seq reveals that glioblastoma recapitulates a normal neurodevelopmental hierarchy. Nat Commun. Nature Publishing Group; 2020;11(1):3406-19. https://doi.org/10.1038/s41467-020-17979-8

58. Long S, Li G. Comprehensive analysis of a long non-coding RNA-mediated competitive endogenous RNA network in glioblastoma multiforme. Exp Ther Med. Spandidos Publications; 2019;18(2):1081-90. https://doi.org/10.3892/etm.2019.7647

59. Fisher DG, Price RJ. Recent Advances in the Use of Focused Ultrasound for Magnetic Resonance Image-Guided Therapeutic Nanoparticle Delivery to the Central Nervous System. Front Pharmacol. Frontiers; 2019;10:1348. https://doi.org/10.3389/fphar.2019.01348

60. Arvanitis CD, Ferraro GB, Jain RK. The blood-brain barrier and blood-tumour barrier in brain tumours and metastases. Nat Rev Cancer. Nature Publishing Group; 2020;20(1):26-41. https://doi. org/10.1038/s41568-019-0205-x

61. Cross DAE, Ashton SE, Ghiorghiu S, Eberlein C, Nebhan CA, Spitzler PJ, et al. AZD9291, an irreversible EGFR TKI, overcomes T790M-mediated resistance to EGFR inhibitors in lung cancer. Cancer Discov. 2014;4(9):1046-61. https://doi.org/10.1158/2159-8290.CD-14-0337

62. Colclough N, Chen K, Johnström P, Fridén M, McGinnity DF. Building on the success of osimertinib: achieving CNS exposure in oncology drug discovery. Drug Discov Today. 2019;24(5):1067-73. https://doi.org/10.1016/j.drudis.2019.01.015

63. Hambardzumyan D, Parada LF, Holland EC, Charest A. Genetic modeling of gliomas in mice: new tools to tackle old problems. Glia. 2011;59(8):1155-68. https://doi.org/10.1002/glia.21142

64. Fomchenko EI, Holland EC. Mouse models of brain tumors and their applications in preclinical trials. Clin Cancer Res. 2006;12(18):5288-97. https://doi.org/10.1158/1078-0432.CCR-06-0438 
65. Hambardzumyan D, Amankulor NM, Helmy KY, Becher OJ, Holland EC. Modeling Adult Gliomas Using RCAS/t-va Technology. Transl Oncol. 2009;2(2):89-95. https://doi.org/10.1593/tlo.09100

66. Tabata H, Nakajima K. Efficient in utero gene transfer system to the developing mouse brain using electroporation: visualization of neuronal migration in the developing cortex. NSC. 2001;103(4): 865-72. https://doi.org/10.1016/S0306-4522(01)00016-1

67. LoTurco J, Manent J-B, Sidiqi F. New and improved tools for in utero electroporation studies of developing cerebral cortex. Cereb Cortex. 2009;19 Suppl 1(suppl 1):i120-5. https://doi.org/10.1093/ cercor/bhp033

68. Zuckermann M, Hovestadt V, Knobbe-Thomsen CB, Zapatka M, Northcott PA, Schramm K, et al. Somatic CRISPR/Cas9-mediated tumour suppressor disruption enables versatile brain tumour modelling. Nat Commun. 2015;6(1):7391. https://doi.org/10.1038/ncomms8391

69. Kawauchi D, Ogg RJ, Liu L, Shih DJH, Finkelstein D, Murphy BL, et al. Novel MYC-driven medulloblastoma models from multiple embryonic cerebellar cells. Oncogene. 2017;36(37):5231-42. https:// doi.org/10.1038/onc.2017.110

70. Patel SK, Hartley RM, Wei X, Furnish R, Escobar-Riquelme F, Bear H, et al. Generation of diffuse intrinsic pontine glioma mouse models by brainstem-targeted in utero electroporation. Neuro Oncol. 2020;22(3):381-92.

71. Glasgow SM, Zhu W, Stolt CC, Huang T-W, Chen F, LoTurco JJ, et al. Mutual antagonism between Sox10 and NFIA regulates diversification of glial lineages and glioma subtypes. Nat Neurosci. 2014;17(10):1322-9. https://doi.org/10.1038/nn.3790

72. Zhu W, Krishna S, Garcia C, Lin C-CJ, Mitchell BD, Scott KL, et al. Daam2 driven degradation of VHL promotes gliomagenesis. Elife. 2017;6:474. https://doi.org/10.7554/eLife.31926

73. Yu K, Lin C-CJ, Hatcher A, Lozzi B, Kong K, Huang-Hobbs E, et al. PIK3CA variants selectively initiate brain hyperactivity during gliomagenesis. Nature. 2020;578(7793):166-71. https://doi.org/10.1038/ s41586-020-1952-2

74. Warren KE. Diffuse intrinsic pontine glioma: poised for progress. Front Oncol. 2012;2:205. https:// doi.org/10.3389/fonc.2012.00205

75. Robison NJ, Kieran MW. Diffuse intrinsic pontine glioma: a reassessment. J Neurooncol. 2014;119(1): 7-15. https://doi.org/10.1007/s11060-014-1448-8

76. Buczkowicz P, Bartels U, Bouffet E, Becher O, Hawkins C. Histopathological spectrum of paediatric diffuse intrinsic pontine glioma: diagnostic and therapeutic implications. Acta Neuropathol. 2014;128(4):573-81. https://doi.org/10.1007/s00401-014-1319-6

77. Mackay A, Burford A, Carvalho D, Izquierdo E, Fazal-Salom J, Taylor KR, et al. Integrated Molecular Meta-Analysis of 1,000 Pediatric High-Grade and Diffuse Intrinsic Pontine Glioma. Cancer Cell. 2017;32(4):520-5.

78. Pathania M, De Jay N, Maestro N, Harutyunyan AS, Nitarska J, Pahlavan P, et al. H3.3K27M Cooperates with Trp53 Loss and PDGFRA Gain in Mouse Embryonic Neural Progenitor Cells to Induce Invasive High-Grade Gliomas. Cancer Cell. 2017;32(5):684-9. https://doi.org/10.1016/j.ccell.2017.09.014

79. Bender S, Tang Y, Lindroth AM, Hovestadt V, Jones DTW, Kool M, et al. Reduced H3K27me3 and DNA hypomethylation are major drivers of gene expression in $\mathrm{K} 27 \mathrm{M}$ mutant pediatric high-grade gliomas. Cancer Cell. 2013;24(5):660-72. https://doi.org/10.1016/j.ccr.2013.10.006

80. Mohammad F, Weissmann S, Leblanc B, Pandey DP, Højfeldt JW, Comet I, et al. EZH2 is a potential therapeutic target for H3K27M-mutant pediatric gliomas. Nat Med. 2017;23(4):483-92. https://doi. org/10.1038/nm.4293

81. Cordero FJ, Huang Z, Grenier C, He X, Hu G, McLendon RE, et al. Histone H3.3K27M Represses p16 to Accelerate Gliomagenesis in a Murine Model of DIPG. Mol Cancer Res. 2017;15(9):1243-54. https://doi.org/10.1158/1541-7786.MCR-16-0389

82. Miklja Z, Yadav VN, Cartaxo RT, Siada R, Thomas CC, Cummings JR, et al. Everolimus improves the efficacy of dasatinib in PDGFR $\alpha$-driven glioma. J Clin Invest. 2020;130(10):5313-25. https://doi. org/10.1172/JCI133310

83. Ruben JD, Dally M, Bailey M, Smith R, McLean CA, Fedele P. Cerebral radiation necrosis: incidence, outcomes, and risk factors with emphasis on radiation parameters and chemotherapy. Int J Radiat Oncol Biol Phys. 2006;65(2):499-508. https://doi.org/10.1016/j.ijrobp.2005.12.002 
84. Connell PP, Hellman S. Advances in radiotherapy and implications for the next century: a historical perspective. Cancer Research. American Association for Cancer Res. 2009;69(2):383-92. https://doi. org/10.1158/0008-5472.CAN-07-6871

85. Pollom EL, Fujimoto D, Wynne J, Seiger K, Modlin LA, Jacobs LR, et al. Phase 1/2 Trial of 5-Fraction Stereotactic Radiosurgery With 5-mm Margins With Concurrent and Adjuvant Temozolomide in Newly Diagnosed Supratentorial Glioblastoma: Health-Related Quality of Life Results. Int J Radiat Oncol Biol Phys. 2017;98(1):123-30. https://doi.org/10.1016/j.ijrobp.2017.01.242

86. Romanelli P, Conti A, Pontoriero A, Ricciardi GK, Tomasello F, De Renzis C, et al. Role of stereotactic radiosurgery and fractionated stereotactic radiotherapy for the treatment of recurrent glioblastoma multiforme. Neurosurg Focus. American Association of Neurological Surgeons; 2009;27(6):E8. https://doi.org/10.3171/2009.9.FOCUS09187

87. DeNunzio NJ, Yock TI. Modern Radiotherapy for Pediatric Brain Tumors. Cancers. Multidisciplinary Digital Publishing Institute; 2020;12(6):1533. https://doi.org/10.3390/cancers12061533

88. Breneman JC, Donaldson SS, Constine L, Merchant T, Marcus K, Paulino AC, et al. The Children's Oncology Group Radiation Oncology Discipline: 15 Years of Contributions to the Treatment of Childhood Cancer. Int J Radiat Oncol Biol Phys. 2018;101(4):860-74. https://doi.org/10.1016/j. ijrobp.2018.03.002

89. Eaton BR, Yock TI. Radiation for pediatric low-grade gliomas: who will benefit and how late is soon enough? Neuro Oncol. 2020;22(8):1068-9. https://doi.org/10.1093/neuonc/noaal44

90. Merchant TE, Conklin HM, Wu S, Lustig RH, Xiong X. Late effects of conformal radiation therapy for pediatric patients with low-grade glioma: prospective evaluation of cognitive, endocrine, and hearing deficits. J Clin Oncol. American Society of Clinical Oncology; 2009;27(22):3691-7. https://doi. org/10.1200/JCO.2008.21.2738

91. Merchant TE, Hua C-H, Shukla H, Ying X, Nill S, Oelfke U. Proton versus photon radiotherapy for common pediatric brain tumors: comparison of models of dose characteristics and their relationship to cognitive function. Pediatr Blood Cancer. 2008;51(1):110-7. https://doi.org/10.1002/pbc.21530

92. Greenberger BA, Pulsifer MB, Ebb DH, MacDonald SM, Jones RM, Butler WE, et al. Clinical outcomes and late endocrine, neurocognitive, and visual profiles of proton radiation for pediatric low-grade gliomas. Int J Radiat Oncol Biol Phys. 2014;89(5):1060-8. https://doi.org/10.1016/j.ijrobp.2014.04.053

93. Zureick AH, Evans CL, Niemierko A, Grieco JA, Nichols AJ, Fullerton BC, et al. Left hippocampal dosimetry correlates with visual and verbal memory outcomes in survivors of pediatric brain tumors. Cancer. 3rd ed. John Wiley \& Sons, Ltd; 2018;124(10):2238-45.

94. Suit H, Urie M. Proton beams in radiation therapy. JNCI J Natl Cancer Inst. 1992;84(3):155-64. https://doi.org/10.1093/jnci/84.3.155

95. Saeed AM, Khairnar R, Sharma AM, Larson GL, Tsai HK, Wang CJ, et al. Clinical Outcomes in Patients with Recurrent Glioblastoma Treated with Proton Beam Therapy Reirradiation: Analysis of the MultiInstitutional Proton Collaborative Group Registry. Adv Radiat Oncol. 2020;5(5):978-83. https://doi. org/10.1016/j.adro.2020.03.022 
